\title{
Control of Spring Back for Helically Coiled Steam Generator Tube
}

Kim Yong Wan ${ }^{1)}$, Kim Jong In $^{1)}$, Chang Moon Hee ${ }^{1)}$, and Suh Yeong Sung ${ }^{2)}$

1) Korea Atomic Energy Research Institute, PO Box 105, YuSong, Taejon, 305-600, Korea

2) Hannam University, 133 Ojung-dong, Taeduk, Taejon, 306-791, Korea

\begin{abstract}
A study has been performed to control the amount of the spring back for tubes of helical steam generator, which is to be installed in the integral reactor. The analytical calculation was done on the basis of beam theory and elasticperfectly plastic material property. The analysis model has considered the effect of the plastic hardening and the effect of the tensile force on the spring back phenomena. Apart from the analytical approach, finite element analysis was carried out to investigate methodological comparison and the quantitative evaluation of the spring back. Coiling process was simulated utilizing commercial finite element analysis program ABAQUS. Parametric studies using an analytical model were performed for various design variables of steam generator tubes in order to produce the design data of the helical steam generator. A sensitivity analysis has shown that the low yield strength, the high elastic modulus, the small helix diameter, and the large tube diameter result in a small amount of the spring back. The application of the tensile force has reduced a considerable amount of spring back. However the application of the tensile force may cause ovality and excessive thickness reduction. From the finite element analysis, it has been shown that the application of axial tensile force reaching $34 \sim 58 \%$ of the tube yield strength is appropriate to satisfy the design criteria of the spring back phenomena, the ovality, and the thickness reduction.
\end{abstract}

\section{INTRODUCTION}

A once-through helical steam generator is being developed to be installed into the integral reactor SMART[1,2]. The spring back phenomena occurring in the coiling process of a steam generator tube induces the dimensional inaccuracy and makes the coiling procedure difficult. The helical tubes are obtained by winding the straight tubes around a rigid mandrel. Tubes of subsequent coils are arranged over the previous coiled tubes. In all cases the residual stresses remaining after the elastic-plastic deformation has taken place during the production process tend to further deform the coiled tube. For physical reasons, the coiled tube geometry must be conserved within very close limits. Therefore, any spring back behavior of the coiled tube should be controlled.

Coiling of titanium tubes has some difficult problems because of the high yield strength to stiffness ratio. Titanium has limited ductility and elongation at room temperatures and titanium tubes have a tendency to neck down in some areas when bent. The non-uniform mechanical and metallurgical properties in the welded region make it difficult to predict coiling performance. It has been reported that tubes smaller than 3 inches in diameter can be satisfactorily coiled without heating[3]. When the tube wall thickness is relatively thin, preheating the tubes is not 
necessary. The plastic stress state subjected to a bending moment has been studied by various researchers[4,5].

The straight tubes are coiled by winding around a rigid mandrel. In all cases the residual stress remaining after the elastic-plastic deformation that took place during the production process tend to further deform the coiled tube. For physical reasons, the coiled tube geometry must be conserved within very close limits. Therefore, any spring back behavior of the coiled tube must be controlled.

A simple analytic equation was derived by $\operatorname{Kim}[6,7]$ based on the beam theory to evaluate the amount of spring back during coiling of the heat transfer tube of a once-through helical steam generator. Based on the analytical model, a parametric study has been carried out for various design variables in order to minimize the absolute amount of spring back in this research. In parallel to the analytical analysis, finite element analysis has been performed to obtain quantitative design data for the amount of the spring back of the helical steam generator tubes. Also, the ovality and the thickness reduction of the tube was investigated.

\section{ANALYTICAL APPROACH}

\section{Analytical Modelling}

The derivation of the analytical model for the sensitivity analysis of the spring back was described in detail in the reference[7]. The spring back analysis model was derived based on the following assumptions. The dimension of the tube cross-section is negligible compared to the length of the tube. The shear deformation of the tube is negligible compared to the bending deformation. The constitutive behavior of the material is considered linear elastic-perfectly plastic or linear hardening. Also, it has been assumed that yielding is determined by the normal stress component in the axial direction of the tube. The helix angle of the coiled tube is assumed to be zero.

If the bending moment $M$ is released after coiling, a circle of radius $R_{o}$ becomes an arc of radius $R_{f}$. Strain can easily be obtained from the stress in a one dimensional model[8]. The residual strain equals the strain of the tube subjected to bending moment $M$ minus the relieved strain during unloading of bending moment. A relation between the radius of curvature before and after unloading of the bending moment can be expressed as follows:

$$
R_{f}=\frac{R_{o}}{1-\frac{R_{o} M}{E I}}
$$

where $E$ denotes Young's modulus and $I$ means moments of inertia. Most of the structural material shows hardening behavior with the progress of plastic deformation. The effect of plastic hardening on the amount of spring back can be evaluated in this model by implementing the linear hardening behavior to the elastic-perfectly plastic model. The tensile force has a strong influence on the amount of spring back in the coiling process of the tube. The material property of the tube was assumed to be elastic-perfectly plastic in this model. The bending moment and the tensile

force are applied to the tube. The application of the tensile force shifts the neutral axis. Subscript $M+F$ denotes that the tube carries the tensile force and bending moment. The shift of the neutral axis $\xi$ can be obtained by force equilibrium and moment equilibrium. For the simplicity of the model, it has been assumed that the tensile force is released first and the bending moment is then released. The moment which determines the amount of the spring back 
of the tube is expressed as follows :

$$
\left.M\right|_{M}=\left.M_{o}\right|_{M}-\left.M_{i}\right|_{M}
$$

where

$$
\begin{aligned}
& \left.M_{o}\right|_{M}=\sigma_{Y} r_{o}^{3}\left[\frac{1}{3}\left(\cos ^{3} \beta+\cos ^{3} \beta_{1}^{\prime}\right)-\frac{1}{3}\left\{\frac{(\alpha+\xi-\delta)(1+\eta)}{2 \alpha-\delta}-1\right\}\left(\cos ^{3} \beta_{2}-\cos ^{3} \beta_{1}^{\prime}\right)\right. \\
& \left.-\frac{(1+\eta)}{32(2 \alpha-\delta)}\left\{\sin 4 \beta_{2}+\sin 4 \beta_{1}^{\prime}\right\}-4\left(\beta_{2}-\beta_{1}^{\prime}\right)\right] \\
& \left.M_{i}\right|_{M}=\sigma_{Y} r_{i}^{3}\left[\frac{1}{3}\left(\cos ^{3} \gamma_{2}+\cos ^{3} \gamma_{1}^{\prime}\right)-\frac{1}{3}\left\{\frac{(\alpha+\xi-\delta)(1+\eta)}{2 \alpha-\delta}-1\right\}\left(\cos ^{3} \gamma_{2}-\cos ^{3} \gamma_{1}^{\prime}\right)\right. \\
& \left.-\frac{(1+\eta)}{32(2 \alpha-\delta)} \frac{r_{i}}{r_{o}}\left\{\sin 4 \gamma_{2}+\sin 4 \gamma_{1}^{\prime}\right\}-4\left(\gamma_{2}-\gamma_{1}^{\prime}\right)\right] \\
& \beta_{1}^{\prime}=\sin ^{-1}(\alpha+\xi-\delta) \\
& \beta_{2}=\sin ^{-1}(\alpha-\xi) \\
& \gamma_{1}^{\prime}=\sin ^{-1}\left[\frac{r_{o}}{r_{i}}(\alpha+\xi-\delta)\right] \\
& \gamma_{2}=\sin ^{-1}\left[\frac{r_{o}}{r_{i}}(\alpha-\xi)\right]
\end{aligned}
$$

$r_{i}$ and $r_{o}$ are the inner and outer radius of the tube, respectively. $\sigma_{y}$ is the yield strength. The maximum stress occurs at $\mathrm{y}=r_{o}$ and $a$ means the elastic range. When a equals one or larger than one, there is no residual plastic deformation after unloading of the bending moment. In order to coil the tube, a should be less than one. The radius of curvature after spring back can be obtained by inserting the equation (2) into the equation(1). In order to simplify the equation, it has been assumed that the tube is thin. The force equilibrium along the tube axis can be expressed as follows:

$$
F=\sigma_{Y} r t\left\{\pi-2 \sin ^{-1}(1-2 \xi)\right\}
$$

When the bending moment and the tensile force are applied to the tube, the bending moment necessary to bend the tube can be expressed as follows:

$$
\left.M\right|_{M+F}=2 \sigma_{Y} r^{2} t\left\{\left(\cos \beta_{1}+\cos \beta_{2}\right)+\frac{\xi}{\alpha}\left(\cos \beta_{1}-\cos \beta_{2}\right)+\frac{1}{2 \alpha}\left(\beta_{1}+\beta_{2}\right)-\frac{1}{4 \alpha}\left(\sin 2 \beta_{1}+\sin 2 \beta_{2}\right)\right\}
$$

The force equilibrium in the direction of the tube axis should become zero after the release of the tensile force.

$$
\frac{\pi}{2}(\eta-1)+\left(\frac{\xi}{\alpha}+1\right) \beta_{1}^{\prime}+\left(\frac{\xi}{\alpha}-\eta\right) \beta_{2}+\frac{1}{\alpha}\left(\cos \beta_{1}^{\prime}-\cos \beta_{2}\right)=0
$$

The residual moment after unloading of the tensile force can be expressed as follows:

$$
\left.M\right|_{M}=2 \sigma_{Y} r^{2} t\left\{\left(\cos \beta_{1}^{\prime}+\eta \cos \beta_{2}\right)+\frac{\xi}{\alpha}\left(\cos \beta_{1}^{\prime}-\cos \beta_{2}\right)+\frac{1}{2 \alpha}\left(\beta_{1}^{\prime}+\beta_{2}\right)-\frac{1}{4 \alpha}\left(\sin 2 \beta_{1}^{\prime}+\sin 2 \beta_{2}\right)\right\}
$$

The curvature of the tube can be obtained by inserting the equation(12) into the equation(1). 


\section{Parametric Calculations}

By using the derived model and the equations, a parametric study has been carried out for various design variables of the steam generator heat transfer tubes. The helical steam generator was designed using titanium alloy tubes. The yield strength of the titanium tube is $350 \mathrm{MPa}$ and the elastic modulus is $117 \mathrm{GPa}$. The coiling radius was assumed to be $100 \mathrm{~mm}$ and the tube inner diameter and outer diameter were assumed to be $9 \mathrm{~mm}$ and $12 \mathrm{~mm}$ respectively. The absolute value of spring back increases as the yield strength increases. That is, the amount of spring back is large for high yield strength material although high yield strength is recommended from the view point of strength. The amount of the spring back was smaller for the higher value of elastic modulus. However, the elastic modulus is not a controllable design variable after the selection of the material.

The effect of plastic hardening on the amount of spring back was investigated utilizing the elastic-linear plastic material properties. The amount of spring back increases as the plastic tangent stiffness increases. If the plastic tangent stiffness, $E_{T}$, becomes zero, the model becomes elastic-perfectly plastic. If the plastic tangent stiffness approaches $E$, it becomes linear elastic. The radius of the curvature at the final configuration was calculated for various radii of curvature of the mandrel. When the other design variables are fixed, the amount of spring back was larger for a larger value of mandrel radius. The effect of the tube diameter on the amount of the spring back has been investigated. As the tube diameter increases, the amount of spring back decreases. If the mandrel radius is fixed, a large plastic deformation is expected for a large tube diameter. The amount of spring back was calculated for various thicknesses of the tubes with a fixed outer diameter. The tube thickness has little influence on the amount of spring back compared to the other geometric design variables. A reduction of tube thickness can diminish only a small amount of spring back. The tube thickness is not a controllable variable from the view point of spring back since it is determined by the strength criteria.

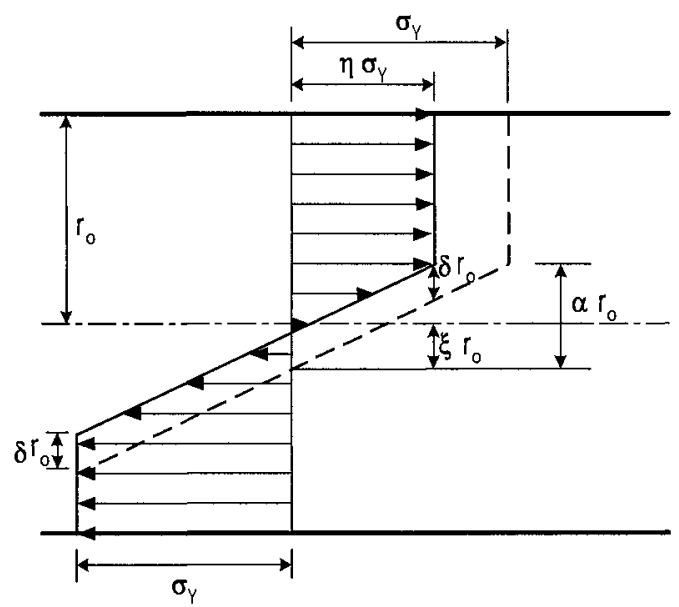

Fig. 1 Description of 1-dimensional model.

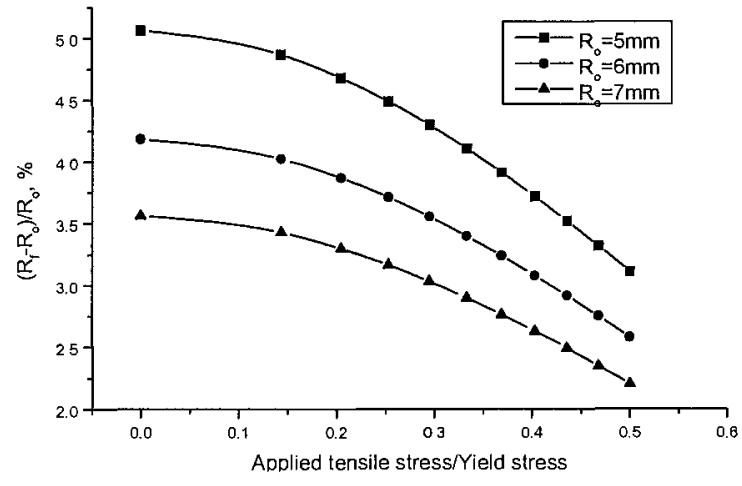

Fig. 2 Effect of tensile force on the spring back

The application of tensile force in the process of coiling is an effective method to reduce the amount of the 
spring back. The effect of the tensile force on the amount of spring back was studied in Fig. 2 where the shift of neutral axis $\xi$ denotes the amplitude of tensile force indirectly applied to the tube. As the tensile force increases, the spring back has decreased. The amplitude of the tensile force to be applied in the coiling process should be determined considering all the other design variables.

A sensitivity analysis has shown that a low yield strength, high elastic modulus, a small helix diameter, and large tube diameter induce small amount of spring back. The amount of spring back can be controlled by the selection of an adequate design value in the basic design stage. It can be reduced to the allowable limit by the application of tensile force to the tube in the coiling stage.

\section{FINITE ELEMENT ANALYSIS}

Finite Element Modelling

Finite element analysis has been performed for the coiling process of steam generator tube. ABAQUS/Standard shell element has been used for the analysis. Large deformation and large rotation has been considered in the analysis. The coiling procedure was simulated by rotating the roller where the end of the tube is fixed. As shown in Fig. 3, half of the tube was modelled because of the symmetry. After the finite element analysis, the radius of curvature obtined from the circle defined by the three points 1 of a,b,c of Fig.4. The ovality of the tube is the average value of the ovality at point $a, b, c$.

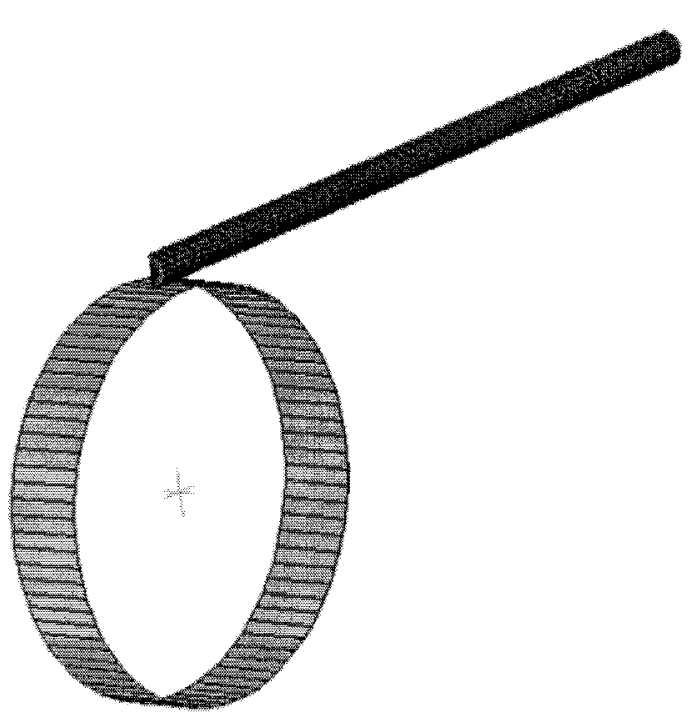

Fig. 3 Finite element model
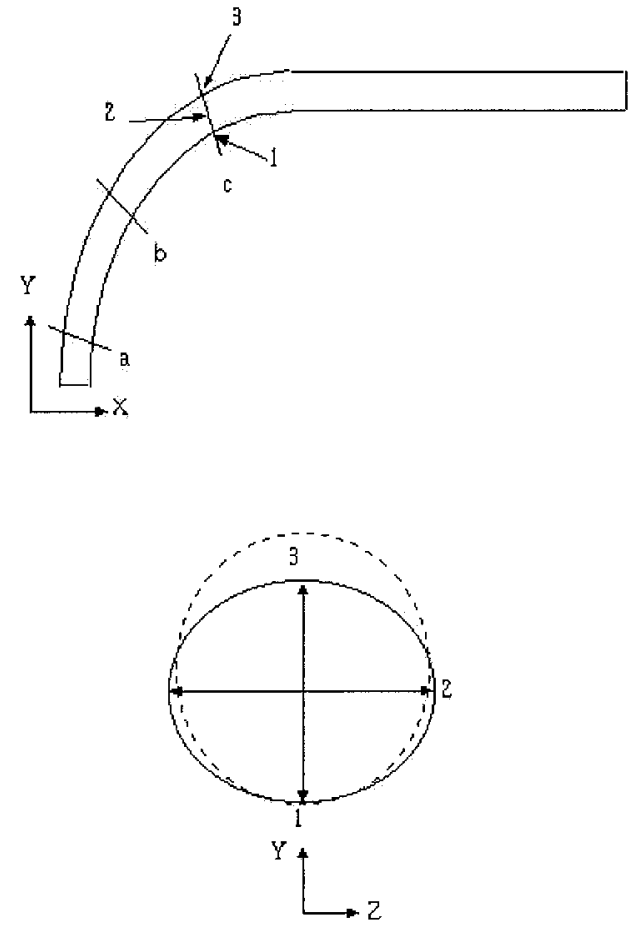

Fig. 4 Determination of radius of curvature 
Finite Element Analysis

In the simulation of coiling process without application of axial tensile force, the axial stress distributions before spring back and after spring back were shown in Fig. 5 and Fig.6. The difference between these two cases causes the spring back. A large axial stress reaching 414MPa is shown before spring back but approximately half of the axial stress was released during the spring back.

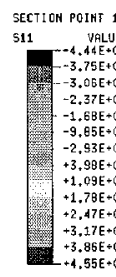

Fig. 5 Axial stress before spring back(no tensile force)

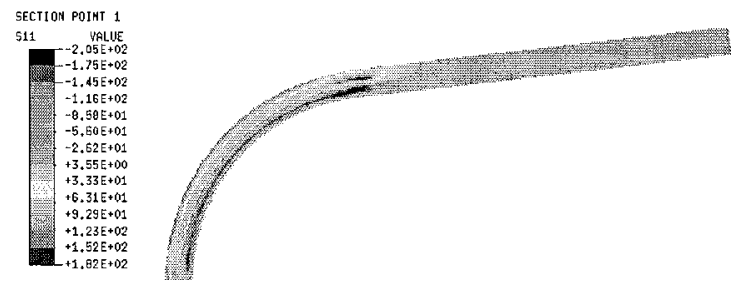

Fig. 6 Axial stress after spring back (no tensile force)

In order to investigate the effect of tensile force, the same simulation has been carried out with the application of the axial tensile force reaching $20 \%$ of the yield stress. As shown in Fig. 7 and Fig. 8 , the maximum axial stress was reduced to $231 \mathrm{MPa}$ before spring back and 167MPa after spring back. The amount of axial stress difference, which determines the amplitude of the spring back was only 64MPa. Without the application of the tensile force, the stress difference was 239MPa. However, the application of tensile force has increased the amount of thickness reduction. The distribution of the tube wall thickness was shown in Fig. 9 and Fig.10. When tensile force was not applied, minimum thickness was $1.44 \mathrm{~mm}$. However the application of tensile force has reduced the minimum thickness. As shown in Fig. 11, the increase of axial tensile force has reduced the amount of spring back but it has increased the amount of ovality and thickness reduction. According to ASME, the ovality should not exceed $8 \%$. For the convenience of the manufacturing procedure and dimensional accuracy, the spring back should not exceed $3 \mathrm{~mm}$. In the view point of tube strength and structural reliability, thickness reduction should not exceed $5 \%$ of the original thicknesss. It has been shown that axial tensile load reaching to $34 \sim 58 \%$ of yield stress should be applied to the tube during the coiling process in order to satisfy all these conditions.
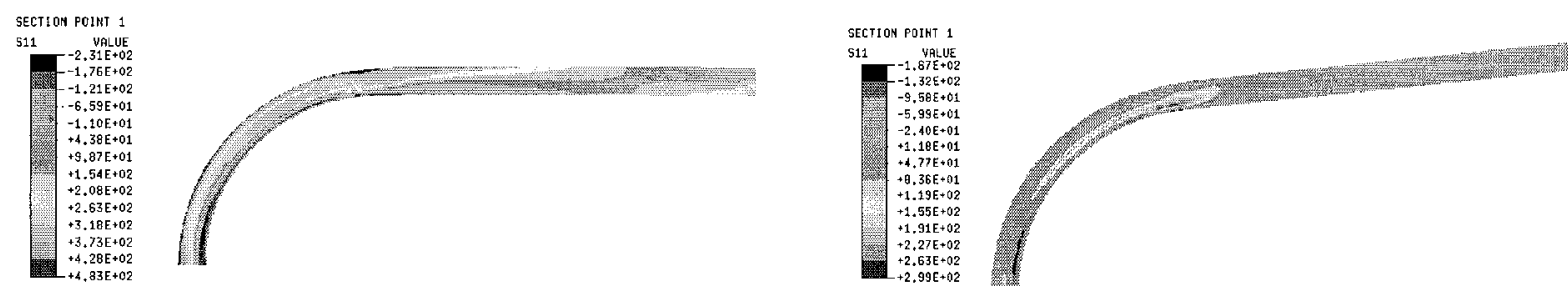

Fig. 7 Axial stress before spring back $\left(0.2 \sigma_{y}\right.$ tensile force $)$ Fig. 8 axial stress after spring back $\left(0.2 \sigma_{y}\right.$ tensile force $)$ 

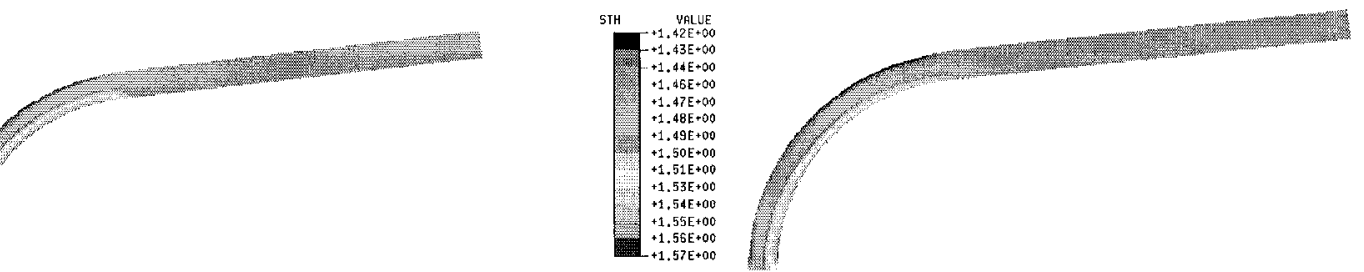

Fig. 9 Thickness reduction(no tension)

Fig. 10 Thickness reduction $\left(0.2 \sigma_{y}\right.$ tensile force $)$

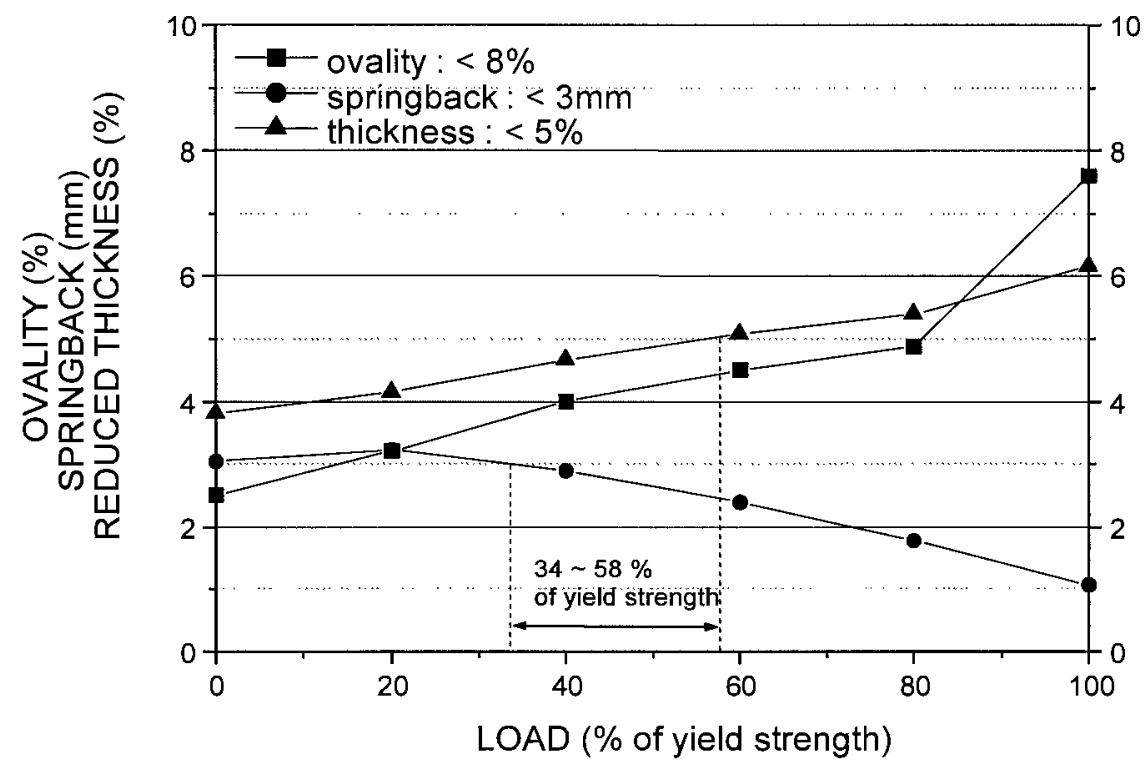

Fig. 11 Effect of tensile force on the spring back, thickness reduction, and ovality.

\section{CONCLUSION}

A comparative study has been performed for the spring back phenomina which occur during the coiling process of helical steam generator tube. A sensitivity analysis has shown that a low yield strength, high elastic modulus, a small helix diameter, and large tube diameter induce a small amount of spring back. The amount of spring back can be controlled by the selection of an adequate design value in the basic design stage. It can be reduced to the allowable limit by the application of tensile force to the tube in the coiling stage. Application of tensile force during the coiling process reduces the amount of spring back. However, excessive tensile force can induce the ovality and tube thickness reduction. From the finite element analysis, it has been shown that the application of axial tension reaching 34 58\%of the yield strength is appropriate to resolve the spring back phenomena, ovality, and the thickness reduction.

Acknowledgement : This work has been performed under the nuclear research \& development program sponsored by MOST. 


\section{REFERENCE}

[1] Y. W. Kim, D. J. Lee, and M. H. Chang, "Analysis of Design Characteristics of Integral PWR as an Advanced Reactor," Journal of Korean Nuclear Society, Vol.27, NO.2, 1995, pp.269-279.

[2] J. I. Kim, et al., Development and Verification Test of Integral Reactor Major Components, KAERI/RR-1889/98, 1999.

[3] L. L. Kollmorgen, "How to Bend Titanium Tubing," The Tool and Manufacturing Engineer, April 1964, pp.90-92.

[4] K.E. Buck and J.W. Hammel, "Fabrication Effects on the Structural Behavior of Large Coils for Fusion Experiments," Nuclear Engineering and Design, Vol.54, 1979, pp.225-238.

[5] G. Li and M.N. Berton, "Cyclic Behavior of a Pipe Section Subjected to Bending, Ovalization or Torsion in the Case of a Perfectly Plastic Material," International Journal of Pressure Vessels \& Piping, Vol.54, 1993, pp.363-386.

[6] Y. W. Kim et al., Development of Spring Back Analysis Model for the SMART Steam Generator Tube, KAERI/TR-1406/99. 1999.

[7] Y.W. Kim and J.I. Kim, "An analysis to minimize the amount of spring back after coiling of helical steam generator tubes," International Journal of Pressure Vessels and Piping (In press).

[8] S. Timoshenko and J.N. Goodier, Theory of Elasticity, 2nd Ed., McGraw-Hill, NewYork 1951. 